\title{
Hardening Curve Plotting and Determination of the Coating Limiting State Criterion based on the Continuous Indentation Method and Computational Modelling
}

\author{
Alexander Ivanchenko ${ }^{1}$, Alexey Zhdanov ${ }^{1}$ and Valentin Morozov ${ }^{1}$ \\ ${ }^{1}$ Department of the mechanical engineering, Vladimir State University, Gorky str. 87, Vladimir, Russia
}

\begin{abstract}
The article describes the method of determining the parameters of the hardening curve of homogeneous and structured coatings (modulus of elasticity, tangential modulus of elasticity and yield strength) required for the strength analysis of parts with coatings that determine their basic performance and strength characteristics. The dependence for determination of tangential (tangential) modulus of elasticity using the results of nanoindentation is proposed. An algorithm for determining the yield strength of the coating material is developed based on solving the problem of elastic-plastic deformation of the coating during the introduction of the indenter by the finite element method. According to the proposed method, the parameters of the TiN/AlN in coating hardening curve were determined based on the results of indentation of the sample of tool high-speed steel with the appropriate coating.
\end{abstract}

\section{Introduction}

The hardening curve is an important characteristic of the material properties. Based on this curve, we can find the material mechanical characteristics and give an estimation of the stress-strain state during the simulation of the load application process of the components. To determine the hardening curve and standard test methods, such as tension, compression or torsion of the samples, have been mainly used. In many cases, the surface layers determine the main performance characteristics of the coated parts. The strength analysis realization for such parts is impossible without considering the physical and mechanical properties and the coatings structure, whose determination possibilities by using standard test methods are very limited.

Currently, to determine the hardness and modulus of elasticity of the surface layers, the method of continuous indentation under very small loads is increasingly being used, which has been called nanoindentation (NI) $[9,11,12]$. This method, based on the penetration of the indenter into the product surface, in contrast to the classical determination of the hardness number according to the impression mark, uses an indentation diagram which represents the penetration force dependence $P$ on the penetration depth of the indenter $h$. The grounding of this method was developed in the USSR, where it was better known as the kinetic hardness method [10].

\section{Materials and methods}

It is possible to obtain information on both the hardness and the modulus of elasticity during NI, and also to estimate the proportion of the elastic component of the total deformation or elastic recovery $\mathrm{R}=\left(\mathrm{h}_{\mathrm{m}}-\mathrm{h}_{\mathrm{f}}\right) / \mathrm{h}_{\mathrm{m}}$, where $h_{m}$ is the maximum depth of the immersion, $h_{f}$ is the depth after removing the load. The NI method is indispensable for studying thin films and multilayer structures.

To observe the impressions of the multiphase materials, nanoindenters are often equipped with AFMheads, which have a resolution of several nanometres. Fig. 1 illustrates the general diagram of the applicationremoval of the load and the fixed parameters from which are measured $\mathrm{H}=\mathrm{P}_{\max } / \mathrm{A}, \mathrm{S}=\mathrm{dP} / \mathrm{dh}$, the magnitude of the modulus of elasticity "film+indenter" system $E^{*}=S / 2(\pi / A)^{0,5}$ and the magnitude of the elastic afterwork $R=\left(h_{\max }-h_{f}\right) / h_{\max }$, where $\mathrm{A}$ is the area of the impression projection, determined from the depth of maximum penetration of the indenter $h_{\max }$. On the other hand the

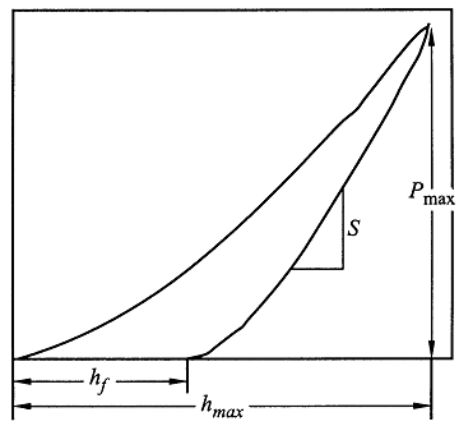

Figure 1. Graphic representation of the load-penetration depth dependencies of the indenter during the load application and the load removal. 
modulus of elasticity of the $\mathrm{E}_{\text {film }}$ is determined from the correlation $1 / E^{*}=\left(1-v_{\text {ind }}{ }^{2}\right) / E_{\text {ind }}+\left(1-v_{\text {film }}{ }^{2}\right) / E_{\text {film }}$, where $v_{\text {ind }}{ }^{2}$ and $v_{\text {film }}{ }^{2}$ are the Poisson's ratios, according to the indenter and the film $\left(v_{\text {film }}{ }^{2} \sim 0,2\right)$ and $E_{\text {ind }}$ is the modulus of elasticity of the indenter (for the diamond $\mathrm{E}=1141 \mathrm{GPa}$, $v=0,07)$

The mechanical properties of the nanocomposite coatings are defined by their hardness $H$; effective Young's modulus $E^{*}=E /\left(1-v^{2}\right)$ and elastic afterwork (recovery) $W_{e}$; here $E$ is the Young's modulus and $v$ the is Poisson's ratio. These magnitudes can be calculated from the load/unload curves that were measured by the micro (nano) hardness tester. The dependences $H=f\left(E^{*}\right)$, $H^{3} / E^{* 2}=f(H), W_{e}=f(H)$ are the main correlations which describe the mechanical properties of the thin films.

In order to solve the problem of elasto-plastic deformation during the strength analysis of the parts with a coating, factors of safety calculation and use of durability models, it is necessary to determine a number of parameters for the schematization of the hardening curve for the coating material. To determine the hardening curve parameters (modulus of elasticity $\mathrm{E}$, Poisson's ratio $v$, tangential modulus of elasticity $E_{\text {tan }}$ and yield limit $\sigma_{0,2}$ of the coating's material), it is proposed to use the following algorithm:

- to execute the indentation for the two load application levels $\mathrm{p}_{1}$ and $\mathrm{p}_{2}$, obtaining the following data: $\mathrm{h}_{\max 1}, \mathrm{~h}_{\max 2}$ (penetration under the loads $\mathrm{p}_{1}$ and $\mathrm{p}_{2}$ respectively), $\mathrm{h}_{\mathrm{f} 1}, \mathrm{~h}_{\mathrm{f} 2}$ (penetration after removal the loads $\mathrm{p}_{1}$ and $\mathrm{p}_{2}$ ), modulus of elasticity E and Poisson's ratio v;

- to determine the values of the introduced volumes of the indenter $\Delta \mathrm{V}_{\max 1}, \mathrm{~V}_{\max 2}, \mathrm{~V}_{\mathrm{f} 1}$ and $\mathrm{V}_{\mathrm{f} 2}$ for $\mathrm{h}_{\max 1}$, $\mathrm{h}_{\max 2}, \mathrm{~h}_{\mathrm{f} 1}$ and $\mathrm{h}_{\mathrm{f} 2}$ respectively;

- to determine the tangential modulus of elasticity $\mathrm{E}_{\mathrm{tan}:}:$

$$
E_{\text {tan }}=\frac{\frac{E}{3(1-2 v)}\left(\left(\Delta V_{\max 2}-\Delta V_{f 2}\right)-\left(\Delta V_{\max 1}-\Delta V_{f 1}\right)\right)}{\Delta V_{\max 2}-\Delta V_{\max 1}}
$$

- to determine the yield limit $\sigma_{0,2}$ of the coating's material by performing a computer simulation of the impression process of the indenter in an elastoplastic setting for different yield limit values $\sigma_{0,2}$ having verified the adequacy of the simulation results according to the experimental data..

During the solution of the elastoplastic problem, the choice of the yield criterion, flow rule and the hardening rule (law) influences on the correctness of the obtained results. Considering that the load increases monotonically during the simulation of the indenter penetration process, and we do not calculate the load removal process, so the required accuracy can be provided by the linear isotropic hardening model, which is applicable to isotropic materials and under considerable deformations the models with kinematic hardening are preferable. The equations of the plastic flow theory establish a relation between the infinitesimal increments of the strains and stresses to the stresses themselves and certain parameters of the plastic state. According to this theory, the relative change in volume for an isotropic body is small, and it represents the elastic deformation $\varepsilon$, which is proportional to the average pressure $\sigma$

$$
\varepsilon=3 k \sigma, k=(1-2 v) / E
$$

or

$$
d \varepsilon=3 k d \sigma
$$

The total increments of the deformations components $d \varepsilon_{i j}$ are made up of the increments of the elastic deformation $d \varepsilon_{i j}^{e}$ and the plastic deformation $d \varepsilon_{i j}^{p}$ components

$$
d \varepsilon_{i j}=d \varepsilon_{i j}^{e}+d \varepsilon_{i j}^{p}
$$

The increments of the elastic deformation components are related to the increments of the stresses components according to the Hooke's law

$$
d \varepsilon_{i j}^{e}=\frac{1}{2 G}\left(d \sigma_{i j}-\frac{3 v}{1+v} \delta_{i j} d \sigma\right)
$$

where $\delta_{i j}$ is the Kronecker symbol;

$\mathrm{G}$ is the shear modulus.

The stress deviator $D_{\sigma}$ and the increment deviator of the plastic deformation $D_{d \varepsilon}^{p}$ are proportional, i.e.

$$
D_{d \varepsilon}^{p}=d \lambda D \sigma
$$

where $d \lambda$ is a small scalar multiplier.

According to the experiments, the increments of the plastic deformation components are proportional to the stresses at a given instant of time. In other words, the stressed state determines the momentary component increments during the plastic deformation. From this, it results the following relations:

$$
d \varepsilon_{i j}^{p}=d \lambda S_{i j}, S_{i j}=\sigma_{i j}-\delta_{i j} \sigma
$$

Calculating now the increment of the plastic deformation work, we find:

$$
d A_{p}=\sigma_{i j} d \varepsilon_{i j}^{p}=d \lambda \sigma_{i j} S_{i j}=2 d \lambda T^{2}
$$

where $\mathrm{T}$ is the intensity of the tangential stresses.

Thus, the factor $d \lambda$ is related to the magnitude of the increment of the plastic deformation work; as $d A_{p} \geq 0$, and $d \lambda \geq 0$. For the total increments of the deformation tensor components, we obtain the expression:

$$
d \varepsilon_{i j}=d \varepsilon_{i j}^{e}+d \lambda S_{i j}
$$

where the increments of the elastic deformation components should be taken according to the Hooke's law. These equations have been proposed by Prandtl and Reis. If the components of the elastic deformation are passed up in the Prandtl-Reis equations, which are permissible under an advanced plastic deformation, so we use the 
Saint-Venant-Mises equations of the plasticity theory. In general, the equations are not complete, since they contain an unknown factor $\mathrm{d} \lambda$, and to determine this factor, it is necessary to have an additional correlation, for example, the Mises yield condition. The yield condition is written in the form:

$$
\phi=\sigma_{e q}-\sigma_{0,2}
$$

Here, $\sigma_{\text {eq }}$ is the scalar, which is equivalent to the tension calculated according to the components of the stress tensor and $\sigma_{0,2}$ is the reference magnitude, yield limit. If the yield of flow does not depend on the rate of deformation, at $\phi<0$ the material remains elastic and at $\phi=0$ the plastic deformations arise in it. For viscoplastic materials, the yield limit can be a function of the strains rate.

The equivalent stresses according to the Mises criterion are determined by the formula

$$
\sigma_{e q}=\frac{1}{\sqrt{2}} \sqrt{\left(\sigma_{1}-\sigma_{2}\right)^{2}+\left(\sigma_{2}-\sigma_{3}\right)^{2}+\left(\sigma_{1}-\sigma_{3}\right)^{2}}
$$

The yield begins when the condition $\sigma_{\mathrm{eq}}=0,2$ is satisfied, where $\sigma_{0,2}$ is the yield limit at an uniaxial stress.

Then $d \lambda=d A_{p} /\left(2 T^{2}\right)$, i.e., the factor is proportional to the increment of the plastic deformation work. If the Mises condition is satisfied, $d T=0$ and the plastic deformation occurs. If $d T=0$, the medium leaves the yield state and starts the load removal, proceeding according to the Hooke's law.

We now take as an additional correlation the condition of the isotropic hardening

$$
d A_{p}=\Phi(T) d T
$$

Denoting $\Phi^{\prime}(T) /\left(2 T^{2}\right)=F(T)$, we obtain

$$
d \lambda=F(T) d T
$$

Thus,

$$
d \varepsilon_{i j}=d \varepsilon_{i j}^{e}+F(T) d T S_{i j}
$$

These correlations are valid when $d T=0$. We note that in case of hardening, the obtained correlations establish a single valued dependence of the increments of the strain components on the stresses and their increments.

Following the proposed algorithm, it is possible to obtain the required combination of parameter values to

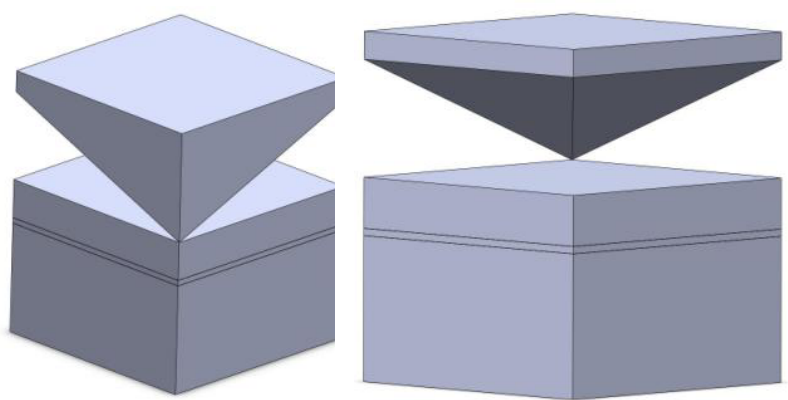

Figure 2. 3D model of an indenter and a coated sample schematize the hardening curve of the material coating. The indenter impression simulation is performed by the finite element method in an elastoplastic setting.

The determination of the physical and mechanical characteristics of the surface layer was carried out based on the results of the indentation of a sample from the tool high-speed steel P6M5 with a TiN/AIN hardening coating with a $3,5 \mu \mathrm{m}$ high on a Ti substrate with a $0,5 \mu \mathrm{m}$ high. The indentation was carried out with a diamond-tipped tool shaped like a tetrahedral pyramid with an angle between the faces of 136 degrees according to the Vickers method by using a microindenter from CSM Instruments. Two series of measurements were carried out with loads of $0,1 \mathrm{~N}$ and $0,05 \mathrm{~N}$. The indentation results used in the further processing are given in Table 1.

Table 1. Indentation results of a P6M5 steel sample with a TiN/AlN coating

\begin{tabular}{|c|c|c|c|c|}
\hline $\begin{array}{c}\text { Load, } \\
\mathrm{H}\end{array}$ & $\begin{array}{c}\text { Penetration } \\
\text { under load, } \\
\mathrm{h}_{\max }, \mu \mathrm{m}\end{array}$ & $\begin{array}{c}\text { Penetration after } \\
\text { load removal, } \\
\mathrm{h}_{\mathrm{f}}, \mu \mathrm{m}\end{array}$ & $\begin{array}{c}\text { Reduced } \\
\text { modulus of } \\
\text { elasticity, } \\
\mathrm{GPa}\end{array}$ & $\begin{array}{c}\text { Hardness, } \\
\mathrm{HV}\end{array}$ \\
\hline 0,05 & 0,315 & 0,148 & 302,7 & 2390 \\
\hline 0,1 & 0,517 & 0,35 & 297,2 & 1960 \\
\hline
\end{tabular}

Having assumed a Poisson's ratio of $v=0,3$ for the coating and having averaged the indentation results, we have obtained a modulus of elasticity value of $E=273 \mathrm{GPa}$. Reference data were used for the substrate material and the P6M5 steel, namely:

substrate $v=0,36 ; \mathrm{E}=96 \mathrm{GPa}$;

steel P6M5 v=0,31; E=203 GPa.

Having determined the volumes of the penetrating part of the indenter corresponding to hmax and hf, the tangential modulus of elasticity Etan $=140 \mathrm{GPa}$ was calculated for the coating material from the obtained dependence (1).

To determine the yield limit of the coating material, it was necessary to simulate the penetration of an indenter into an elastoplastic material using program complexes of finite element analysis.

The penetration simulation of the indenter into the coating material was carried out using the ANSYS software in a three-dimensional setting. Fig. 2 shows the 3-d model created in the SolidWorks simulation environment and Fig. 3 shows the finite element model used for the calculations.
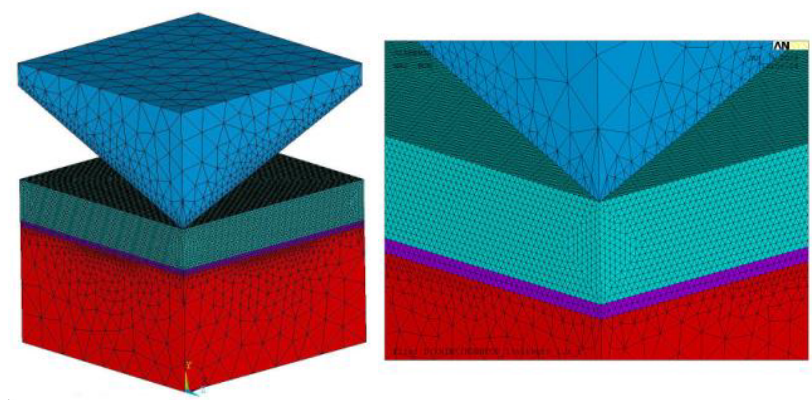

Figure 3. Finite element model of an indenter and a coated sample 


\section{Results}

From the symmetry conditions of the model's geometry and the load, a $1 / 4$ part of the indenter and the sample was considered; thereafter a $1 / 4$ part of the load was applied. To plot the finite element mesh, an eight-node hexagonal element was chosen. The coating was modelled by using 16 layers of elements and the substrate by two layers. The geometric dimensions of the simulated sample were chosen so that the plastic deformation did not reach the lateral boundaries of the sample. The contact problem setting corresponded to the contact diagram of an absolutely rigid body with a deformable one, where the surface of the rigid body is always the target one, and the surface of the deformable body is the contact one. The problem was solved using the Newton-Raphson procedure and the frontal direct solver. The diamond indenter was considered as a linearly elastic isotropic material with a Young's modulus of $\mathrm{E}=1140 \mathrm{GPa}$ and a Poisson's ratio of $v=0,07$. During the solution of the elastoplastic penetration problem, a model of linear isotropic hardening was used, which is applicable to isotropic materials and under considerable deformations the models with kinematic hardening are preferable. In this model, the Mises yield condition is used in conjunction with the equations of the Prandtl-Reiss flow theory. During isotropic hardening, the yield surface is expanded uniformly in all directions; in addition, it is assumed that the yield limit on tension and compression due to hardening increases identically, that is, the Bauschinger effect in this model is not taken into account. Since we examine a single load application, the nature of the hardening of the material could not affect the results of the calculation. Fig. 4 presents the calculation results of the indenter displacement under a load of $0,1 \mathrm{~N}$ and Fig. 5 presents the strained state of the sample surface during the indenter penetration.

After having carried out multivariate calculations with

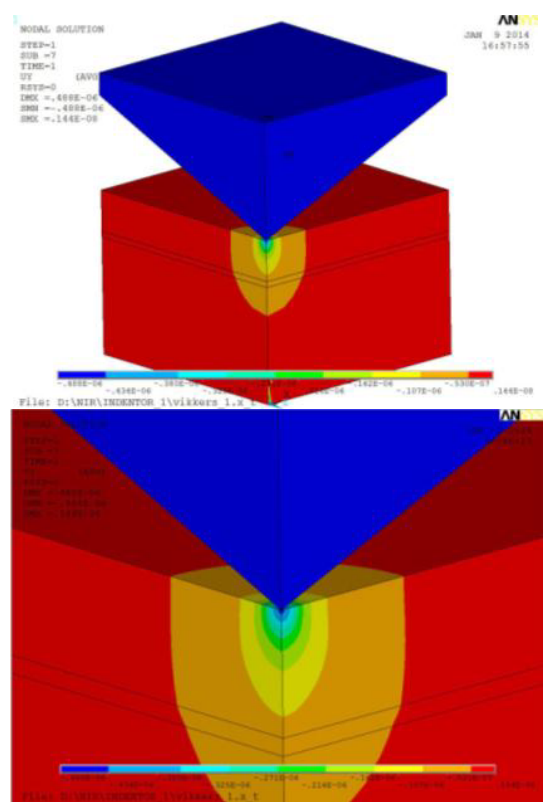

Figure 4. Nodal displacements field during the indenter penetration under a load of $0,1 \mathrm{~N}$, the specified yield limit of the coating material is $\sigma_{0,2}=3700 \mathrm{MPa}$

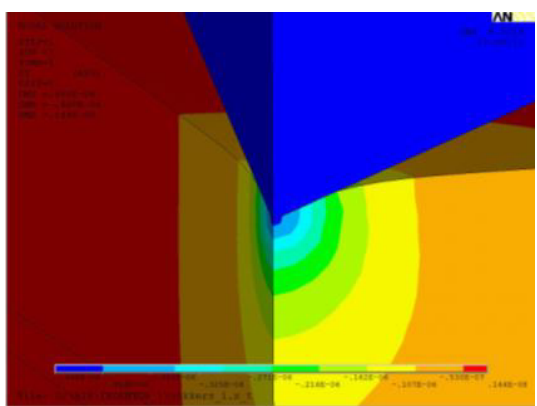

Figure 5. Strained state of the sample surface during the indenter penetration under a load of $0,1 \mathrm{~N}$, the specified yield limit of the coating material is $\sigma_{0,2}=3700 \mathrm{MPa}$, the size factor $\mathrm{K}_{\mu}=1$

different values of the yield limit of the coating material under loads of 0,1 and $0,05 \mathrm{~N}$, the minimum relative errors were obtained at $\sigma_{0,2}=3700 \mathrm{MPa}$. The results are shown in Table. 2

Table 2. Simulation results of the indentation process of a sample of P6M5 steel coated with TiN/AIN at a given yield limit of the coating material $\sigma_{0,2}=3700 \mathrm{MPa}$

\begin{tabular}{|c|c|c|c|}
\hline Load, $\mathrm{H}$ & $\begin{array}{c}\text { Penetration of the } \\
\text { indenter during } \\
\text { the test, } \\
\mathrm{h}_{\max }, \mu \mathrm{m}\end{array}$ & $\begin{array}{c}\text { Calculated } \\
\text { penetration of the } \\
\text { indenter, } \\
\mathrm{h}_{\max }, \mu \mathrm{m}\end{array}$ & $\begin{array}{c}\text { Relative } \\
\text { error,\% }\end{array}$ \\
\hline 0,05 & 0,315 & 0,321 & 1,9 \\
\hline 0,1 & 0,517 & 0,488 & 4,9 \\
\hline
\end{tabular}

\section{Conclusions}

The obtained results of the calculation are consistent with the characteristics of high-speed steel P6M5K5 after heat treatment, namely: the yield limit during compression $\sigma_{0,2}=3100 \mathrm{MPa}$ and the hardness $\mathrm{HV}=885$ [14].

\section{References}

1. Yu. A. Golovin, Nanoindentation and mechanical properties of solids in submicrovolumes, thin nearsurface layers, and films, Physics of the Solid State, 50, 12 (2008), pp. 2116-2142.

2. W. C. Oliver, and G.M. Pharr, Measurement of hardness and elastic modulus by instrumented indentation: Advances in understanding and refinements to methodology, J. Mater. Res. 19, 1 (2004), pp. 3-21.

3. S. A. Fedosov, and L. Peshek, Determining mechanical properties of materials by microindentation: Modern foreign methods, MSU Fac. of Phys. (2004).

4. S. I. Bulychev, V. P. Alekhin, M. Kh. Shorshorov and A. P. Ternovskii, Mechanical properties of materials studied from kinetic diagrams of load versus depth of impression during microimpression, Problemy Prochnosti, 9 (1976), pp. 79-83.

5. K. Johnson, Contact Mechanics, Mir (1989).

6. V. G. Sorokin, A. V. Volosnikova, S. A. Vyatkin et al., Steel and Alloy Grades, Mashinostroenie (1989), 640 p. 